\title{
AC Electric Fields Drive Steady Flows in Flames
}

\section{Citation}

Drews, Aaron M., Ludovico Cademartiri, Michael L. Chemama, Michael P. Brenner, George M. Whitesides, and Kyle J. M. Bishop. 2012. AC Electric Fields Drive Steady Flows in Flames.

Physical Review E 86, no. 3: 036314.

\section{Published Version}

doi:10.1103/physreve.86.036314

\section{Permanent link}

http://nrs.harvard.edu/urn-3:HUL.InstRepos:11931821

\section{Terms of Use}

This article was downloaded from Harvard University's DASH repository, and is made available under the terms and conditions applicable to Open Access Policy Articles, as set forth at http:// nrs.harvard.edu/urn-3:HUL.InstRepos:dash.current.terms-of-use\#OAP

\section{Share Your Story}

The Harvard community has made this article openly available.

Please share how this access benefits you. Submit a story.

Accessibility 


\title{
Manipulating flames at a distance with AC electric fields
}

\author{
Aaron M. Drews ${ }^{1}$, Ludovico Cademartiri ${ }^{3}$, Michael L. Chemama ${ }^{2}$ \\ Michael P. Brenner ${ }^{2,4}$, George M. Whitesides ${ }^{3,4}$, and Kyle J. M. Bishop ${ }^{1 *}$ \\ ${ }^{1}$ Department of Chemical Engineering, Pennsylvania State University \\ University Park, Pennsylvania 16802 \\ ${ }^{2}$ Harvard School of Engineering and Applied Sciences \\ Cambridge, MA 02138 \\ ${ }^{3}$ Department of Chemistry and Chemical Biology, Harvard University \\ Cambridge, MA 02138 \\ ${ }^{4}$ Kavli Institute, Harvard University \\ Cambridge, MA 02138
}

* Address correspondence to kjmbishop@engr.psu.edu

Abstract. We show that time-oscillating electric fields applied to plasmas present in flames create steady flows of gas. Ions generated within the flame move in the field and migrate a distance $\delta$ before recombining; the net flow of ions away from the flame creates a time-averaged force that drives the steady flows observed experimentally. A quantitative model describes the response of the flame and reveals how $\delta$ decreases as the frequency of the applied field increases. Interestingly, above a critical frequency, AC fields can be used to manipulate flames at a distance without the need for proximal electrodes.

The most familiar description of a flame is "a local region of high temperature generated by rapid, exothermic chemical reactions". A flame is, however, also a plasma. In particular, the combustion of hydrocarbon fuels generates sufficient densities of charged species that even small flames (the flame of a humble candle) can be considered as chemically driven, nonequilibrium plasmas [1]. As such, flames interact with external electric fields, which can be used to monitor [2], manipulate [3, 4], and enhance [5, 6] the processes that make up "combustion" through a variety of physical and chemical mechanisms. Sufficiently strong electric fields $\left(>10^{6} \mathrm{~V} / \mathrm{m}\right.$ at atmospheric pressure) influence the chemistry of combustion by accelerating electrons to energies capable of exciting, dissociating, or ionizing neutral species upon impact [6]. Even fields that are too weak to influence combustion directly can cause 
significant hydrodynamic flows [1] - so called electric or ionic winds [7] - through the collisional transfer of momentum from accelerated charged species to the neutral gas $[8,9]$.

Previous efforts to manipulate flame plasmas with electric winds have relied almost exclusively on static (DC) fields [1] to induce steady currents of charged species that drive steady gas flows (although see [4, 10,11]). Despite promising demonstrations (e.g., enhancing flame stability [5], manipulating heat transfer [3], reducing pollutant emissions [12]), the magnitude of these effects - and their applicability - is strictly limited by ionic screening, which requires direct contact of the electrodes and the flame to maximize the ionic currents and gas velocities [8].

Here, we report that time-oscillatory (AC) electric fields also induce steady electric winds in flame plasmas with gas velocities exceeding those due to static fields of comparable magnitude. Furthermore, unlike static fields, the electric force due to AC fields is localized near the surface of the flame. Consequently, the AC response depends only on the local field at the surface of the flame - not on the position of the electrodes used to generate the field. These results suggest that oscillating electric fields can be used to manipulate and control combustion processes at a distance.

To characterize and explain these effects, we investigated a simple experimental system comprising a laminar methane-air flame positioned between two parallel-plate electrodes. We quantify both the electric and hydrodynamic response of the flame as a function of frequency and magnitude of the applied field. A theoretical model shows how steady gas flows emerge from the time-averaged electrical force due to the field-induced motion of ions generated within the flame and by their disappearance by recombination. Our predictions of both the flow velocity and the electric response agree quantitatively with the experimental results.

Experimental. A small methane flame was positioned symmetrically between two parallel aluminum electrodes $(20 \mathrm{~cm} \times 20 \mathrm{~cm})$, separated by a distance $L=10 \mathrm{~cm}$ (Fig. 1a). The burner was a thermally and electrically insulating cylindrical tube (alumina composite, i.d. $6.4 \mathrm{~mm}$, o.d. $9.5 \mathrm{~mm}$ ) through which flowed a mixture of methane, oxygen, and nitrogen (molar composition, $\mathrm{CH}_{4}: \mathrm{O}_{2}: \mathrm{N}_{2}=0.15: 0.17: 0.68$; flow rate $=500 \mathrm{sccm}$; equivalence ratio, $\phi=1.75$, with air as oxidizer). When needed, the nitrogen stream was doped with a fine mist (1-10 $\mu \mathrm{m}$ droplets, created by an ultrasonicator) of $1 \mathrm{M}$ aqueous $\mathrm{NaOH}$ to increase the density of ions within the flame $[13,14]$. To mitigate external air flows, we enclosed the 
flame and the electrodes in a glass box $(30 \times 30 \times 30 \mathrm{~cm})$ with a central, triangular opening $(\sim 20 \mathrm{~cm})$ in its top to vent combustion products.

A high-voltage amplifier (Trek 40/15) applied sinusoidal, time-varying electric fields to the flame. AC fields caused the transient redistribution of charged species generated within the flame and resulted in the formation of two gas jets directed symmetrically outwards towards the two electrodes (Fig. 1b). To characterize the response of the flame, we measured two quantities ( $i$ ) the electric current $I$ through the circuit illustrated in Figure 1a using an analog preamplifier (DL Instruments 1211) interfaced with a data acquisition system (NI-9223), and (ii) the velocity of the field-induced gas flows using high-speed schlieren velocimetry $[15,16]$.

Static Fields. As previously reported [1, 8], the application of a static field to a methane flame creates an electric wind (Fig. 2a shows $E_{\mathrm{o}}=280 \mathrm{kV} / \mathrm{m}$ ). Charge carriers generated within the flame migrate to the respective electrodes, with positive [17] and negative [18] charges moving with and against the direction of the field. The accumulation of charge on both sides of the flame results in an electric force density $f=\rho E$ ( $\rho$ is the charge density, $E$ is the local electric field) that drives two jets of gas in opposite directions. To contrast this DC response with the AC effect described below, we briefly quantify the effect of static electric fields in the experimental set-up used here.

The electric force density $f$ driving the flow is directly proportional to the electric current $I$, which can be measured directly (Fig. 2b); this proportionality holds because the electric force is approximately $f \approx q_{ \pm} n_{ \pm} E$ while the current density is $j \approx q_{ \pm} K_{ \pm} n_{ \pm} E \sim I$, where $q_{ \pm}, n_{ \pm}$, and $K_{ \pm}$are the charge, number density (ions/volume), and mobility (drift velocity/field strength) of the charge carriers. For the methane flame without $\mathrm{NaOH}$ dopant, the current increases quadratically with the field before saturating at a constant value. At low fields, the accumulation of charge on both sides of the flame acts to screen the field in the region of the flame and limits the current. Within the space-charge regions, the ion density is proportional to the applied field $n_{ \pm} \sim E_{o}$, and the current scales as $j \sim E_{o}^{2}[1,8,16]$. For sufficiently strong fields, the current is instead limited by the rate of ionization within the flame - that is, all charged species generated by combustion are removed by the field via the electrodes. By doping the flame with additional ions, the transition from the space-charge-limited regime to the reaction-limited regime can be shifted to larger fields (Fig. 2b). 
In the space-charge-limited regime, the velocities of the gas jets increase linearly with the magnitude of the applied field (Fig. 2c) as explained by Bernoulli's principle [1]. Briefly, the pressure drop is proportional to the electric force $\Delta p \sim f$, which in turn is proportional to the current density $f \sim j$, such that $\Delta p \sim j \sim E_{o}{ }^{2}$. The characteristic velocity is therefore $u=\left(\Delta p / 2 \rho_{f}\right)^{1 / 2}=C E_{o}$, where $\rho_{f}$ is the fluid density and $C$ is a constant. Assuming $\Delta p \approx \varepsilon_{o} E_{o}{ }^{2}$ (cf. below), these scaling arguments suggests $C \approx 3.9 \times 10^{-6} \mathrm{~m}^{2} \mathrm{~V}^{-1} \mathrm{~s}^{-1}-$ as compared to $C=2.9 \times 10^{-6} \mathrm{~m}^{2} \mathrm{~V}^{-1} \mathrm{~s}^{-1}$ determined experimentally (Fig. 2c).

Oscillating Fields. Application of an AC field, $E(t)=\sqrt{ } 2 E_{o} \cos (\omega t)$, to a doped flame creates an electric wind similar to that produced by a static field: two gas jets flow outwards from the flame towards the electrodes (Fig. 1b shows $v=\omega / 2 \pi=1 \mathrm{kHz}$ and $E_{\mathrm{o}}=100 \mathrm{kV} / \mathrm{m}$ ). Because the applied field is symmetric, the jets are nearly identical in shape, and their velocity increases strongly with increasing field strength $E_{o}$ and weakly with increasing frequency $v$ (Fig. 3a). At low frequencies, the gas velocity is well described by the DC relation from Figure 2c. At higher frequencies, the velocity increases by $c a .20 \%$ at the highest frequencies accessible by the amplifier $(\sim 1 \mathrm{kHz})$.

To characterize the electric response of the flame, we calculated the energy dissipation rate $\Phi=\langle I(t) V(t)\rangle$ due to the motion of charged species in and around the flame as a function of frequency and field strength (Fig. 3b), where $I(t)$ is the measured current, $V(t)$ is the applied voltage, and \langle\rangle denotes a time average. In the absence of the flame, the dissipation rate is negligible, as expected for an ideal capacitor; the measured dissipation rate thus characterizes the electric response of the flame itself [16].

At low frequencies, $\Phi$ approaches a constant value corresponding to the oscillation average of the DC current-voltage relationship $I_{d c}(V)$ in Figure $2 \mathrm{~b}$ - that is, $\Phi \approx\left\langle I_{d c}(V(t)) \cdot V(t)\right\rangle$. Using the quadratic fit $I_{d c}=D V^{2}$ from Figure $2 \mathrm{~b}$ with $D=1.1 \times 10^{-14} \mathrm{~A} \mathrm{~V}^{-2}$, the low-frequency dissipation rate is approximately $\Phi \approx(8 \sqrt{2} D / 3 \pi) V_{o}^{3}$ where $V_{o}=L E_{o} \quad\left(e . g\right.$. for $E_{o}=200 \mathrm{kV} / \mathrm{m}, \Phi \approx 0.11 \mathrm{~W}$ in quantitative agreement with experiment; $c f$. Fig. 3b). Above a critical frequency (dashed curve in Fig. 3b), the dissipation rate increases by a factor of $\sim 5$ at the highest frequencies $(\sim 1 \mathrm{kHz})$; this increase suggests a transition in the structure of the flame plasma due to the AC field.

Discussion. Electrohydrodynamic flows are driven by an electric body force of the form $f=\rho E$, which enters into the Navier-Stokes equations governing fluid motion. For simplicity, we consider the onedimensional geometry illustrated in Figure 4a. Gauss's law implies that the electric force can be expressed 
as $f(x, t)=\varepsilon_{o} \partial E^{2}(x, t) / \partial x$. Averaging in time over one period of oscillation gives $\langle f\rangle=\varepsilon_{o} d\left\langle E^{2}\right\rangle / d x$. For the experimental conditions, the field inside the flame is negligible, $\left\langle E^{2}\right\rangle \approx 0$, due to screening by mobile charged species. Far from the flame, the average field strength approaches that of the applied field, $\left\langle E^{2}\right\rangle \approx E_{o}^{2}$. The transition of $\left\langle E^{2}\right\rangle$ from 0 to $E_{o}^{2}$ occurs across some region of size $\delta$ within which the electric force is localized ( $c f$. below). Furthermore, the force is directed outwards from the flame in either direction (i.e., from regions of weak field to regions of strong field in qualitative agreement with the gas jets observed in experiment).

To estimate the gas velocities induced by the steady electric force, we define a characteristic pressure drop $\Delta p \approx \varepsilon_{o} E_{o}^{2}$ by integrating the force from inside the flame to some point far from the flame. Bernoulli's principle then implies a characteristic velocity of $u \approx\left(2 \varepsilon_{o} / \rho_{f}\right)^{1 / 2} E_{o}-$ identical to that derived for static fields.

To determine the length scale $\delta$ over which the force is distributed, we must consider the details of the charge transport in and around the flame. We consider the following model describing the production and consumption of ions within the flame and their transient migration in the field [16].

$$
\begin{gathered}
\frac{\partial n_{ \pm}}{\partial t}+\frac{\partial}{\partial x}\left( \pm K n_{ \pm} E\right)=k_{i} g(x)-k_{r} n_{+} n_{-} \\
\frac{\partial E}{\partial x}=\frac{e}{\varepsilon_{o}}\left(n_{+}-n_{-}\right) \text {where } \int_{0}^{L} E d x=\sqrt{2} L E_{o} \cos (\omega t)
\end{gathered}
$$

Here, the charge carriers are assumed to be monovalent ions with equal mobilities $\pm K \sim 10^{-4} \mathrm{~m}^{2} \mathrm{~V}^{-1} \mathrm{~s}^{-1}$ [19]; the flame is represented by a localized region of ionization $g(x)$ with a characteristic thickness $a \sim 10^{-4} \mathrm{~m}$ (Fig. 4a) and magnitude $k_{i} \sim 10^{20}$ ions $^{-3} \mathrm{~s}^{-1}$ [20]. Ions are consumed through a second-order recombination process with rate constant $k_{r} \sim 10^{-13}$ ions $^{-1} \mathrm{~m}^{3} \mathrm{~s}^{-1}[20]$ or by perfectly absorbing electrodes at the system boundaries. Remarkably, this simple model predicts the average dissipation rate as a function of both frequency and field strength in quantitative agreement with experiments (Fig. 3b solid curves).

Further analysis [16] reveals the length scale over which ions are redistributed by the field: $\delta \sim K E_{o} / \kappa \omega$ where $\kappa=\left(\varepsilon_{o} k_{r} / e K\right)^{1 / 2} \sim 0.3$ is a dimensionless parameter characterizing the relative rates of ion recombination $\left(k_{r} n_{o}\right.$ where $\left.n_{o}=\left(k_{i} / k_{r}\right)^{1 / 2}\right)$ and ion screening $\left(e K n_{o} / \varepsilon_{0}\right)$. Briefly, we rewrite equations (1) 
in terms of the total ion density $n_{s}=n_{+}+n_{-}$and the charge density $n_{d}=n_{+}-n_{-}$, maintaining only the dominant terms.

$$
\begin{gathered}
\frac{\partial n_{s} / \partial}{\partial t}+\frac{\partial}{\partial x}\left(K n_{d} E\right)=k_{j} g(x)-\frac{1}{4} k_{r}\left(n_{s}^{2}-\not p_{d}^{2}\right) \\
\frac{\partial n_{d}}{\partial t}+\frac{\partial}{\partial x}\left(K n_{s} E\right)=0
\end{gathered}
$$

Approximating $\partial / \partial t$ as $\omega, \partial / \partial x$ as $\delta^{-1}$, and field strength by $E_{o}$, equations (2) - (4) yield the characteristic length scale $\delta \sim K E_{o} / \kappa \omega$, ion density $n_{s} \sim \varepsilon_{o} \omega / e K$, and charge density $n_{d} \sim \kappa \varepsilon_{o} \omega / e K$. Examining the magnitude of the neglected terms, this dominant balance governs charge transport outside of the flame (where $g(x) \sim 0$ ) provided $\delta \ll L$ and $\kappa \ll 1$.

From a microscopic perspective, ions generated within the reaction zone migrate a distance $\delta$ under the influence of the field before recombining. The migration of a single ion creates an impulse (force $\times$ time) of order $\delta e / K$, which is transferred to the neutral gas through molecular collisions. Multiplying this impulse by the net flux of ions leaving the ionization region yields the pressure difference $\Delta p$ driving the flow. Using the scaling arguments above, the ion flux $K n_{d} E$ may be approximated as $\omega \kappa \varepsilon_{o} E_{o} / e$ such that $\Delta p \sim \varepsilon_{o} E_{o}^{2}$ in agreement with the macroscopic derivation above.

When $\delta$ is larger than $L\left(\omega \ll K E_{o} / \kappa L\right)$, ions are consumed at the absorbing electrodes, and the dissipation rate is identical to that observed for static fields averaged over one period of oscillation namely, $\Phi_{\text {low }} \approx\left[6 \sqrt{2} L^{3} / \pi(L-b)^{3}\right] A_{f} \varepsilon_{o} K E_{o}^{3}$, where $A_{f}$ is the characteristic area of the flame (needed to convert the results of the 1D model into 3D), and $b$ is the width of the flame (see Fig. 4a). Above a critical frequency (i.e., when $\delta \ll L$ or $\omega \gg K E_{o} / \kappa L$; dashed curve in Fig. 3b), ions are instead consumed via chemical recombination processes within a thin region surrounding the flame (e.g., $\delta \sim 5 \mathrm{~mm}$ for $v=1 \mathrm{kHz}$ and $\left.E_{o}=100 \mathrm{kV} / \mathrm{m}\right)$. In this limit, the average dissipation rate increases to $\Phi_{\text {high }}=(4 \sqrt{2} / \kappa) A_{f} \varepsilon_{o} K E_{o}^{3}$ and no longer depends on the position of the electrodes (i.e., on $\left.L\right)$.

Consequently and in contrast to flows driven by static fields, $\mathrm{AC}$ electric winds do not require electrical contact between the flame and the surrounding electrodes. This independence is illustrated in Figure $4 \mathrm{~b}$, in which the electrodes have been covered with an electrically insulating material. Upon application of a 
static field, charge rapidly accumulates on the insulating surfaces, screens the applied field, and prevents the formation of electric winds. The response to an oscillating field is unchanged.

At even higher frequencies - namely, for $\omega>e K n_{o} / \varepsilon_{o}$ - the field varies faster than the time required for ions to redistribute and screen the field within the flame. No flows are expected at such frequencies. For the flame plasmas described here, $n_{o} \sim 10^{16}-10^{17}$ ions $\mathrm{m}^{-3}[16]$, and electric winds are expected only for frequencies $v<10-100 \mathrm{kHz}$.

Conclusions. Oscillating electric fields interact with the plasmas generated by combustion of methane to create steady flows of gas. In contrast to electrical forces due to static fields, the forces induced by AC fields act directly at the surface of the flame, and do not depend on the position of the electrodes. Importantly, these results provide a basis for the "electrode free" manipulation and control of combustion processes - e.g., by electromagnetic waves. Beyond the symmetric flows detailed here, it should also be possible to achieve directional flows by varying the geometry of the applied field.

Acknowledgements. This work was supported by DARPA under award number W944NF-09-1-0005.

\section{References.}

[1] J. Lawton and F. Weinberg, Electrical Aspects of Combustion (Oxford University Press, London, 1969).

[2] R. C. Waterfall, R. He, N. B. White, and C. M. Beck, Meas. Sci. Technol. 7, 369 (1996).

[3] K. G. Payne and F. J. Weinberg, Proc. Combust. Inst. 250, 316 (1959).

[4] S. H. Won, M. S. Cha, C. S. Park, and S. H. Chung, Proc. Combust. Inst. 31, 963 (2007).

[5] H. C. Jaggers and A. von Engel, Combust. Flame 16, 275 (1971).

[6] S. M. Starikovskaia, J. Phys. D: Appl. Phys. 39, R265 (2006).

[7] M. Robinson, Am. J. Phys 30, 366 (1962).

[8] J. Lawton and F. J. Weinberg, Proc. Combust. Inst. 277, 468 (1964).

[9] M. Rickard, D. Dunn-Rankin, F. Weinberg, and F. Carleton, J. Electrostat. 64, 368 (2006).

[10] M. K. Kim, S. K. Ryu, S. H. Won, and S. H. Chung, Combust. Flame 157, 17 (2010).

[11] M. K. Kim, S. H. Chung, and H. H. Kim, Proc. Combust. Inst. 33, 1137 (2011).

[12] M. Saito, T. Arai, and M. Arai, Combust. Flame 119, 356 (1999).

[13] R. M. Clements and P. R. Smy, J. Appl. Phys. 40, 4553 (1969).

[14] J. W. Daily and C. Chan, Combust. Flame 33, 47 (1978). 
[15] D. R. Jonassen, G. S. Settles, and M. D. Tronosky, Opt. Lasers Eng. 44, 190 (2006).

[16] See Supplemental Material at [URL will be inserted by publisher] for details on (A) shlieren image processing, (B) AC dissipation measurements, (C) Langmuir probe measurements, and (D) the charge transport model.

[17] J. M. Goodings, D. K. Bohme, and C. W. Ng, Combust. Flame 36, 27 (1979).

[18] J. M. Goodings, D. K. Bohme, and C. W. Ng, Combust. Flame 36, 45 (1979).

[19] For ion-doped flames, the symmetry of the gas jets induced by static fields (Fig. 2b) suggests that positive and negative ions have similar mobilities. In general, this is not the case (e.g., for premixed flames with large electron densities or for sooting flames).

[20] A. B. Fialkov, Prog. Energy Combust. Sci. 23, 399 (1997). 


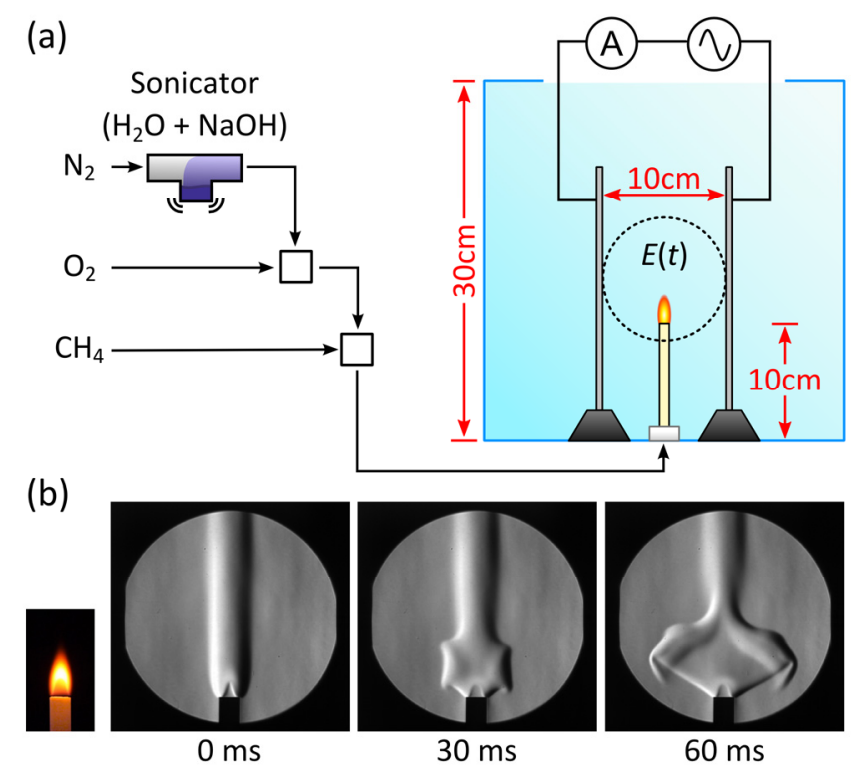

Figure 1. (color online) (a) Schematic illustration of the experimental set-up; the cubic combustion chamber is drawn to scale. The dotted circle illustrates the field of view for the schlieren images below. (b) Schlieren images of a premixed, $\mathrm{NaOH}$-doped methane flame following the application of an oscillating electric field of magnitude $100 \mathrm{kV}_{\mathrm{RMS}} / \mathrm{m}$ at $1 \mathrm{kHz}$. The images are taken at intervals of $30 \mathrm{~ms}$ following the application of the field. 

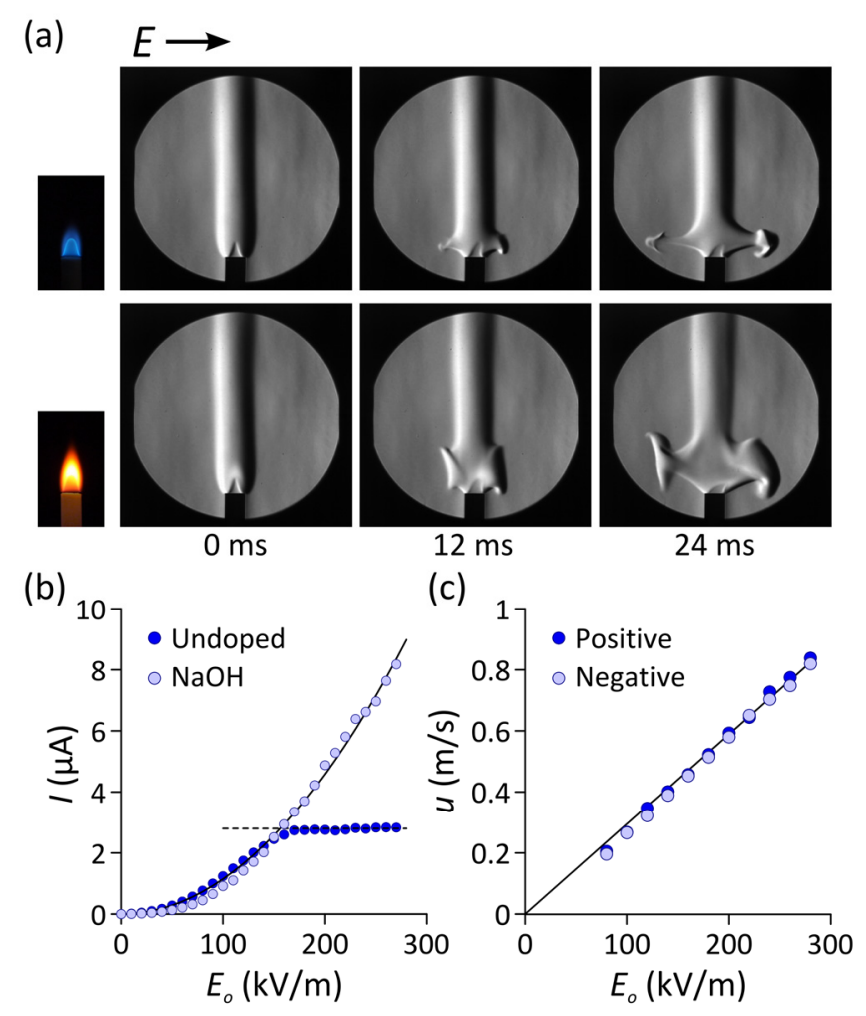

Figure 2. (color online) Static fields. (a) Schlieren images of a premixed methane flame without (top) and with (bottom) $\mathrm{NaOH}$ dopant directly after the application of a static electric field of $280 \mathrm{kV} / \mathrm{m}$. The images are taken at regular intervals of $12 \mathrm{~ms}$. (b) Electric current as a function of the applied field strength for a premixed methane flame with $(O)$ and without ( ) ion doping. The solid and dashed lines illustrate the space charge limited and reaction limited responses, respectively; the fit used for the space charge response is $I=\left(1.1 \times 10^{-16} \mathrm{~A} \mathrm{~m}^{2} \mathrm{~V}^{-2}\right) E_{o}^{2}$. (c) Characteristic gas velocity $u$ as a function of field strength for the $\mathrm{NaOH}$ doped flame; positive $(\bullet)$ and negative $(O)$ refer to the sign of the charged species driving the jet. The solid curve is $u=\left(2.9 \times 10^{-6} \mathrm{~m}^{2} \mathrm{~V}^{-1} \mathrm{~s}^{-1}\right) E_{o}$. 

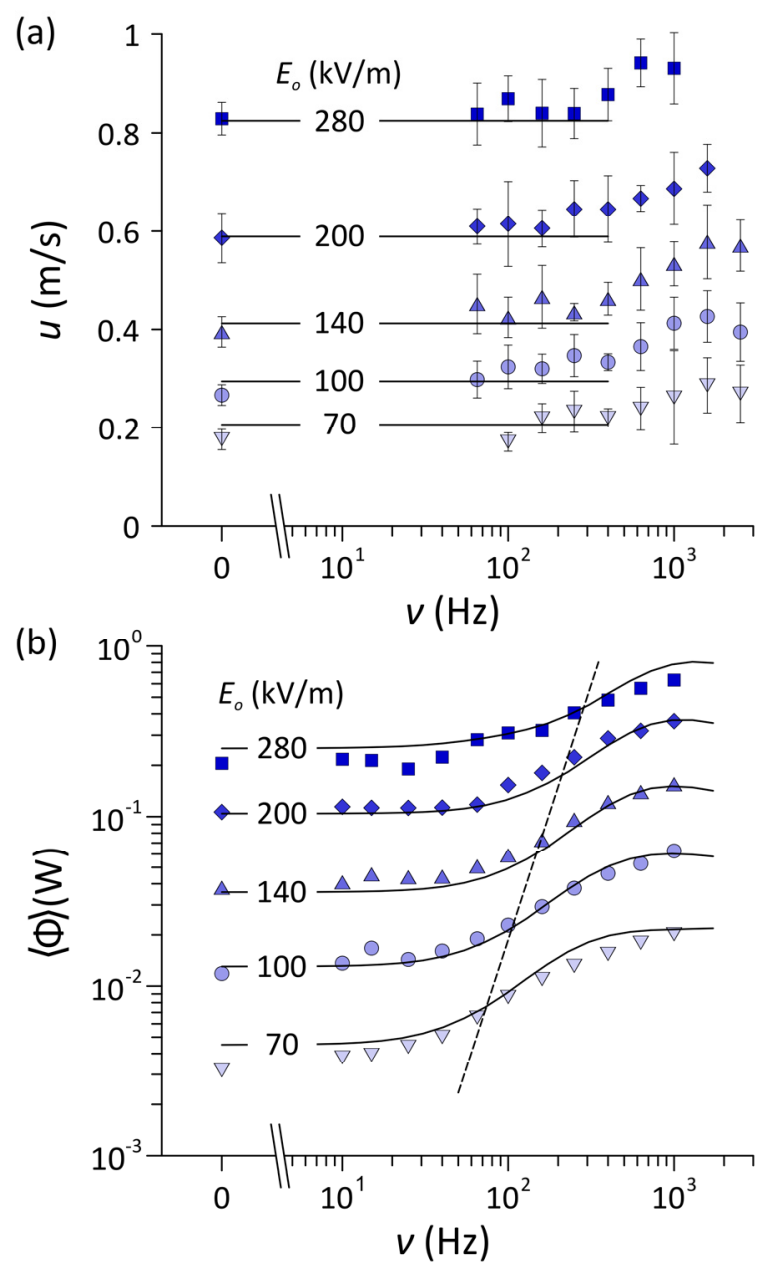

Figure 3. (color online) Oscillating fields applied to $\mathrm{NaOH}$-doped, premixed methane flames. (a) Characteristic gas velocity $u$ as a function of frequency $v$ and field strength $E_{o}$. The solid lines correspond to $u=\left(2.9 \times 10^{-6} \mathrm{~m}^{2} \mathrm{~V}^{-1} \mathrm{~s}^{-1}\right) E_{o}$ as obtained from Figure 2c. (b) Average electric dissipation rate $\Phi$ as a function of frequency $v$ and field strength $E_{o}$. The solid lines are theoretical predictions of the charge transport model. The dashed line illustrates the transition from the low frequency regime to the high frequency regime as defined by the condition $\delta \sim L$. The parameters used in the model are: electrode spacing, $L=0.1 \mathrm{~m}$; flame width, $\quad b=10^{-2} \mathrm{~m} ; \quad$ flame thickness, $a=10^{-4} \mathrm{~m} ; \quad$ mobility, $\quad K=1.05 \times 10^{-4} \mathrm{~m}^{2} \mathrm{~V}^{-1} \mathrm{~s}^{-1}$; recombination rate, $k_{r}=1.90 \times 10^{-13}$ ions $^{-1} \mathrm{~m}^{3} \mathrm{~s}^{-1}$; ionization rate, $k_{i}=1.09 \times 10^{20}$ ions $\mathrm{m}^{-3} \mathrm{~s}^{-1}$; and flame area, $A_{f}=3.90 \times 10^{-3} \mathrm{~m}^{2}$ (see [16] for details). 

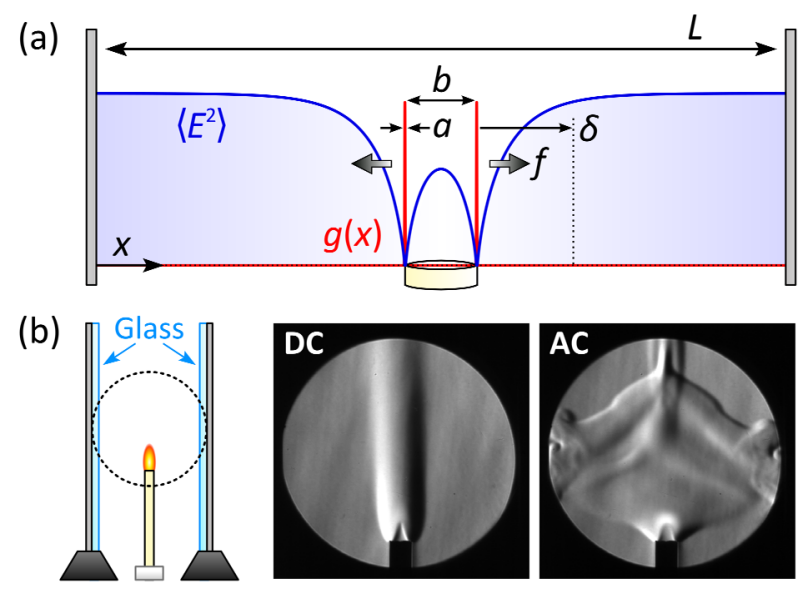

Figure 4. (color online) (a) Schematic illustration of the 1D model showing the ionization region $g(x)$ and the time averaged field squared $\left\langle E^{2}\right\rangle$. (b) Electrohydrodynamic response of flame plasmas using glass-insulated electrodes for static $\left(E_{o}=75 \mathrm{kV} / \mathrm{m}\right.$; left $)$ and oscillatory $\left(E_{o}=75 \mathrm{kV} \mathrm{RMS} / \mathrm{m}, v=800 \mathrm{~Hz} ;\right.$ right $)$ fields. 